\title{
Buckling analysis of a reinforced sandwich column using the Bloch wave theory
}

\author{
Van Dong Do ${ }^{\mathrm{a}}$, Philippe Le Grognec ${ }^{\mathrm{a}, \mathrm{b}, *}$ \\ ${ }^{a}$ Mines Douai, Polymers and Composites Technology 83 Mechanical Engineering Department, 941 rue Charles Bourseul, CS \\ 10838, F-59508 Douai Cedex, France \\ ${ }^{b}$ ENSTA Bretagne, FRE CNRS 3744, IRDL, F-29200 Brest, France
}

\begin{abstract}
This paper deals with sandwich structures whose core layer is made of a homogeneous foam periodically strengthened by orthogonal reinforcements. Beside traditional sandwiches which generally display satisfactory specific flexural properties but fatally insubstantial stiffnesses in the through-thickness direction, 3D reinforced sandwich materials provide optimal out-of-plane mechanical properties. Despite this, buckling remains one of the major failure modes of such structures and, compared to the case of traditional sandwiches, both global and local buckling phenomena are more complicated in presence of transverse reinforcements. Indeed, in most cases, the modal deformed shapes involve simultaneously the skins and the reinforcements in an intricate way. The main feature of these buckling modes is periodicity, but the typical wave length appears to be generally different from the characteristic length between reinforcements. However, it is possible to investigate such periodic modes on a simple unit cell by using the so-called Bloch wave theory. In this work, an efficient procedure is defined so as to deal with the buckling behavior of a sandwich column with periodic orthogonal reinforcements. First, a numerical method is implemented in the framework of the commercial software Abaqus. The evaluation of the critical strains is performed on a unit cell: an initial average compressive strain is enforced, then natural frequencies are computed and the critical strains are deduced by extrapolation of the previous eigenvalues. A Python program is developed so as to automate these successive calculation steps and a Fortran program is also needed (within Abaqus) in order to cope with the two real and imaginary problems to be solved due to the Bloch-periodic conditions. Furthermore, an exact analytical solution of this problem is obtained in the particular case of a reinforced sandwich with no foam core (for simplicity purposes). The analytical and numerical solutions obtained with a unit cell model are finally compared to the results of numerical computations performed on a complete beam with an arbitrary number of cells, for validation purposes. The critical strains/displacements are found to be in very good agreement and the buckling modes rebuilt from the real and imaginary components of the unit cell modal solutions perfectly coincide with the buckling modes of the complete beam obtained through a linearized buckling analysis.
\end{abstract}

Keywords: Sandwich structures, Reinforcements, Global/local buckling, Unit cell, Bloch wave theory, 


\section{Introduction}

Sandwich composites are plate-like structures which traditionally consist of two thin and stiff skin layers separated by a thicker and softer core layer. The core material is often a homogeneous and isotropic foam, which provides the extreme lightweight property of the sandwich. Conversely, the skins and their distance to the middle surface of the composite contribute to the tensile properties and particularly to the flexural rigidity. The resulting composite material thus combines both lightweight and strong mechanical properties and, thanks to this interesting compromise, sandwich structures are increasingly used in aerospace, marine or transportation industries, among others. However, such classical sandwiches show two main weaknesses. The first one concerns the out-of-plane behavior (in through-thickness compression and transverse shear), directly related to the low mechanical properties of the homogeneous soft core material. In order to improve the load carrying capacity in the thickness direction without being detrimental to lightness, the low density core layer may be usually strengthened, using transverse fibrous reinforcements or replacing the foam core by a thin-walled core layer (with a honeycomb or corrugated structure, for instance). Unfortunately, the presence of thin or slender reinforcements adds up to that of thin faces and makes the sandwich material even more sensitive to buckling which turns out to be the major remaining weakness of such composite structures (apart from delamination).

As a matter of fact, due to their geometric and material configuration, sandwich structures are prone to collapse when submitted to compressive loadings. The buckling analysis of sandwich structures is therefore an important issue for dimensioning purposes and so it has been widely studied in the past decades (see [1] and [2] as two of the first leading references in this field). On one hand, when dealing with classical sandwiches, one usually distinguishes two types of geometric instabilities, namely the global buckling of the sandwich structure under overall compression and the so-called wrinkling (or local buckling) of the faces, which may appear insofar as they undergo compressive stresses (when the sandwich structure is submitted to axial compression or pure or simple bending, for instance). If the global buckling of a sandwich material can be easily viewed as the classical buckling of a homogeneous structure (as soon as the equivalent properties have been properly derived), the local buckling analysis requires the use of advanced models. If the earliest contributions rely on uncoupled formulations, where the global and local buckling analyses are treated separately, many authors have tried to achieve unified models capable of describing both global and local modes (both symmetric/hourglass and antisymmetric/snaking) in the particular case of a sandwich column

\footnotetext{
*Corresponding author.

Email address: philippe.le_grognec@ensta-bretagne.fr (Philippe Le Grognec)
} 
under axial compression (Benson and Mayers 3 have been the first to suggest a unified approach to solve the overall buckling and wrinkling problems simultaneously). Among them, a hybrid beam/2D model (with no kinematic assumption in the core layer) was recently developed by Douville and Le Grognec [4] which was first able to derive closed-form expressions of both buckling and wrinkling critical values of sandwich beamcolumns (accounting for all mode types) with a very good accuracy, compared to the numerous simplified models in earlier literature (see also [4] for a comprehensive review of analytical or numerical models for the buckling analysis of sandwich beam-columns/plates under various loading conditions, using different simplified kinematic assumptions).

On the other hand, a compressive loading in the thickness direction of a 3D reinforced sandwich will also fatally lead to an instability phenomenon. This response can be seen as the buckling of reinforcements inside the homogeneous core material, if any. Among others, López Jímenez and Triantafyllidis 5 investigated the buckling behavior of rectangular and hexagonal honeycomb structures under transverse compression, possibly combined with transverse shear. In the context of stitched sandwich structures under transverse compression and out-of-plane shear, the buckling of reinforcements (which can be considered as slender beams) is governed by Euler's theory, as soon as the core material is neglected (see [6], for instance). However, in practice, despite its comparatively low modulus, the presence of the core material cannot be ignored and the classical Euler critical values are no more valid (the core material generally displays a stabilizing effect). Several analytical solutions have thus been proposed in the literature to better estimate the critical loading for such a micro-buckling problem, where simplified strain states are supposed in the core material which is sometimes even replaced by spring distributions [7] (see [8] for a more comprehensive review on this subject). Recently, one of the authors 8 analyzed the buckling behavior of similar composites, namely Napco ${ }^{\circledR}$ reinforced sandwiches (whose manufacturing is based on transverse needling, see Figure 11), under through-thickness compression. Exact closed-form solutions were derived using a similar hybrid beam/2D (unit cell) model as in [4] (where the skins are replaced here by the reinforcements and without any simplification regarding the deformation field in the core material). The prevailing mode was proved to be the so-called shear mode (see Figure 2p, as long as the volume fraction of reinforcements is sufficiently high, as shown in 9 .

Considering now reinforced sandwiches, but under in-plane loading (such as axial compression), most complicated solutions may arise since both faces and reinforcements are supposed to interact in the buckling response. Such sandwich structures are also likely to buckle in a localized as well as in a global way, depending on the geometric and material parameters, but the new modes (and therefore the new critical values) cannot be obtained by a simple combination of the original buckling modes involving either the skins or the reinforcements (see Figure 3 for an illustration of global and local modes of classical or reinforced 


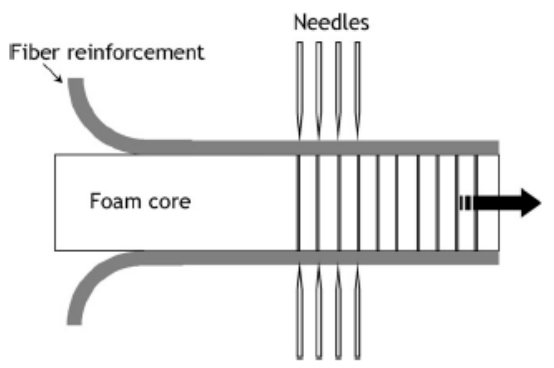

(a) Transverse needling

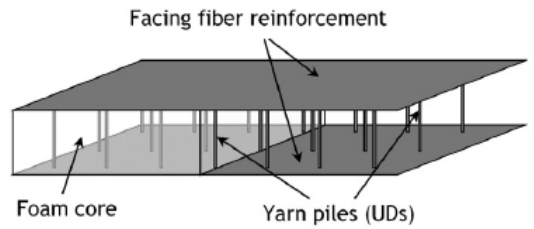

(b) $3 \mathrm{D}$ sandwich representation

Figure 1: Napco ${ }^{\circledR}$ technology

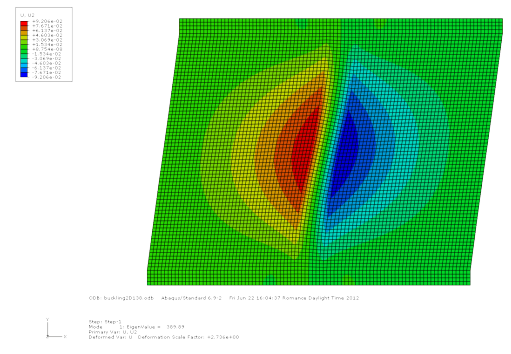

(a) Buckling mode of a 2D unit cell (FE model)

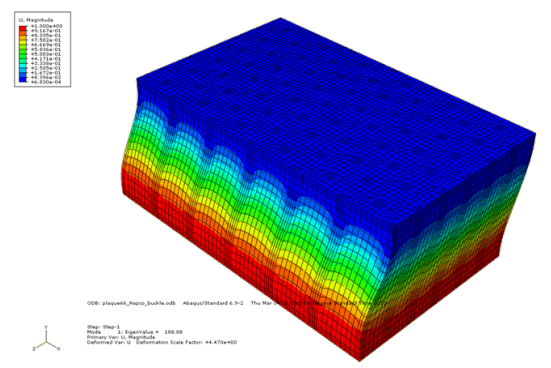

(c) Buckling mode of a 3D structure (FE model)

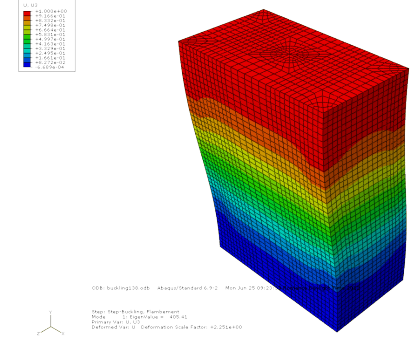

(b) Buckling mode of a 3D unit cell (FE model)

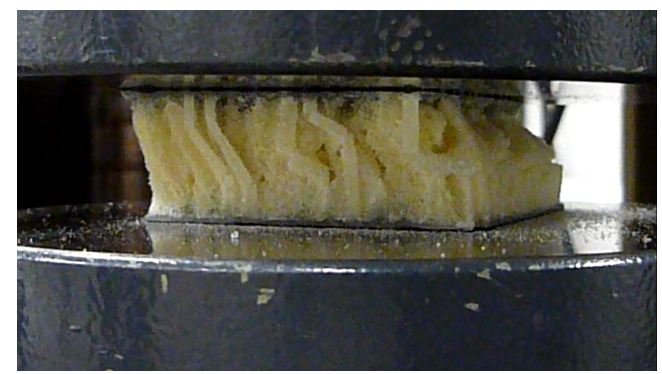

(d) Experimental post-buckled deformed shape

Figure 2: Buckling of a reinforced Napco ${ }^{\circledR}$ sandwich under through-thickness compression 8

sandwiches). Such a problem has thus been far less investigated in the literature. Let us mention Wang and Abdalla [10] who examined the global and local buckling behavior of grid-stiffened composite panels (like sandwiches with only one skin) and Combescure et al. 11] who analyzed the post-bifurcation and stability of an hexagonal honeycomb under equi-biaxial compression, among few others.

The present paper deals again with a sandwich material manufactured with polymeric foam core reinforced thanks to the Napco ${ }^{\circledR}$ technology. Here, this work specially investigates the axial compression behavior of sandwich columns in a 2D context. Based on the previous study dealing with through-thickness compression, a unit cell model is still preferred for obvious efficiency purposes. Two differences occur nonethe- 

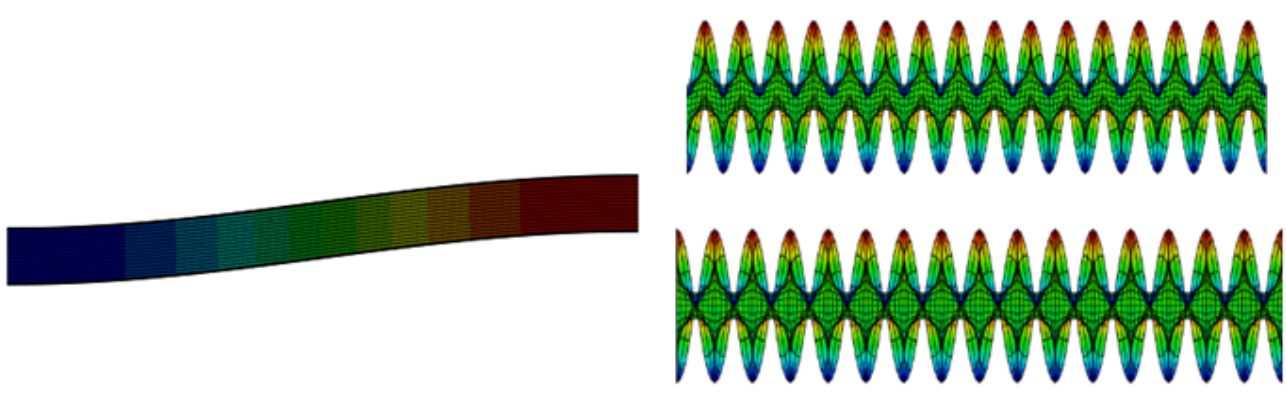

(a) Global mode without reinforcements

(b) Local modes without reinforcements
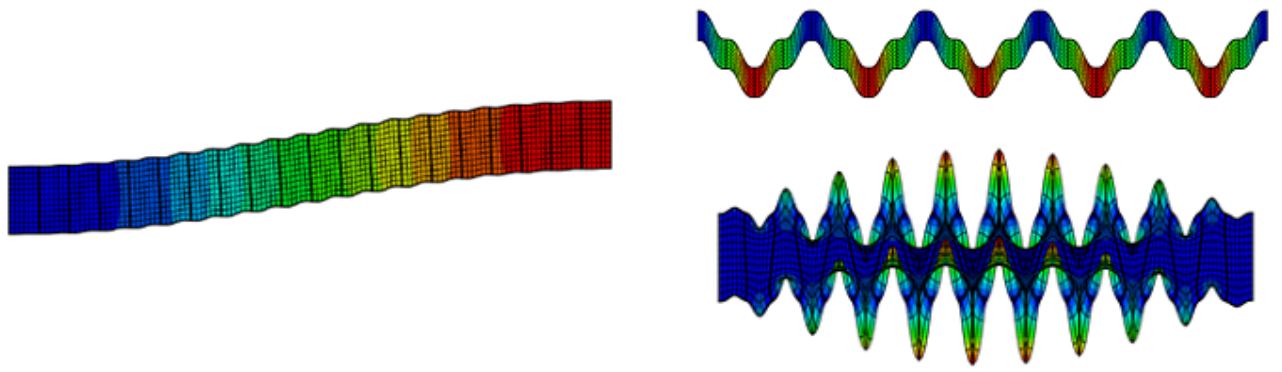

(c) Global mode with reinforcements

(d) Local modes with reinforcements

Figure 3: Influence of the reinforcements on the global and local modal deformed shapes

less between the two problems. Firstly, for the through-thickness compression analysis, the skins were not included in the model, since they did not deform during the corresponding buckling response, whereas the unit cell model here naturally includes both reinforcements and skins. Secondly, periodic boundary conditions were simply applied on the edges of the unit cell, since the wave length of the periodic buckling modes was strictly the length of the unit cell. Here, as seen in Figure 3 , the modes are supposedly periodic but with an arbitrary wave length. An appropriate technique for the identification of such periodic modes using a single unit cell turns out to be the Bloch wave theory. In the sequel, a numerical procedure devoted to the buckling analysis of reinforced sandwich columns under axial compression is first described. It consists of three successive calculation steps, namely (i) the compression of the unit cell with an arbitrary strain, (ii) the evaluation of the natural frequencies and corresponding modes under the so-called Bloch-periodic boundary conditions and (iii) the estimation of the critical strain by means of extrapolation. The presence of complex boundary conditions (with real and imaginary parts) requires the use of a specific Fortran program within the framework of the commercial software Abaqus and a Python program is also implemented so as to automate all the numerical procedure. In the particular case of a reinforced sandwich where the foam core has been removed, analytical solutions are also obtained from a general bifurcation analysis involving the same Bloch-periodic complex conditions. Numerical computations (linearized buckling analyses) on 
complete reinforced sandwich columns (displaying several cells) are finally performed for validation purposes.

\section{Numerical analysis of the buckling behavior of reinforced sandwich columns}

\subsection{Problem definition}

In this study, the objective is to solve numerically (and also analytically) the buckling behavior of a Napco ${ }^{\circledR}$ sandwich under axial compression. The Napco ${ }^{\circledR}$ technology is a manufacturing process of 3D sandwich composites based on transverse needle punching. Among the existing methods, such as tufting, Z-pinning and stitching [12, the patented Napco ${ }^{\circledR}$ technology allows one to produce tailored sandwich structures in a continuous way, while preserving a high production efficiency and a relatively low cost. It consists in strengthening the foam core of a sandwich structure by adding orthogonal (or inclined) throughthickness reinforcements, but it differs from other technologies by the fact that the fibrous reinforcements here come from the skin material, so that the facing fabrics (mats) and the foam core make up a monolithic whole (see Figure 4 .

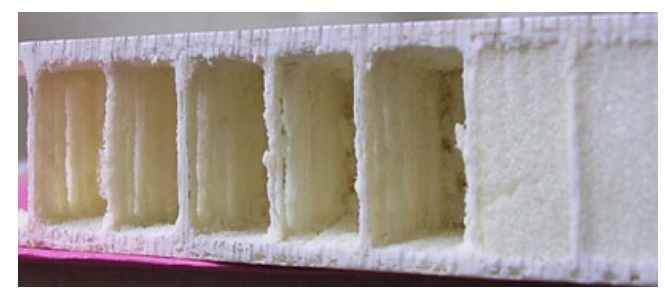

(a) Orthogonal through-thickness reinforcements

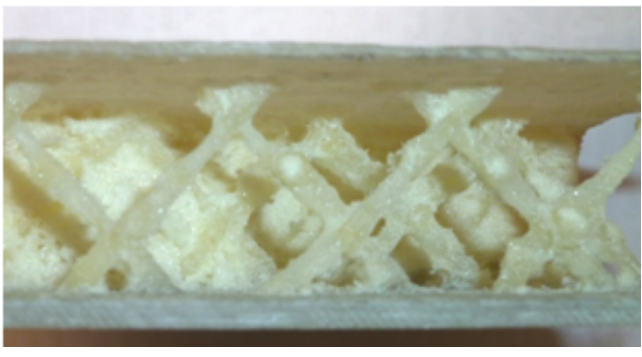

(b) Inclined through-thickness reinforcements

Figure 4: Napco ${ }^{\circledR}$ sandwiches (the foam core is partly removed to show the transverse composite beams)

The core layer is classically made up of a linearly elastic isotropic closed cell polyurethane foam. The skins are also elastic and are supposed to be isotropic, with equivalent Young's modulus $E_{s}$ and Poisson's ratio $\nu_{s}$. The cylindrical fibrous reinforcements (of constant cross-section) are supposed here to be perpendicular to the skins and can be viewed as unidirectional composite columns (UDs) composed of aligned isotropic fibres surrounded by resin. Since the micro-buckling of fibres inside the reinforcements is out of the scope of this paper and thus ignored in our future model, the heterogeneous fibrous reinforcements can simply be represented by equivalent homogeneous cylinders with their effective properties deriving from a preliminary homogenization step, based on advanced mixture laws, for instance. In the present study, only the effective longitudinal modulus will have a significant influence on the results, so that the reinforcements will also be considered isotropic with Young's modulus $E_{f r}$ and Poisson's ratio $\nu_{f r}$. 
From a geometric point of view, a 2D representation of the sandwich column is retained with a unit depth. Some conventions are specially suggested in order to relate properly the 2D designed model to the real 3D configuration. In the context of micro-buckling of a fibrous reinforcement in a surrounding medium, Zhang and Latour 13 . compared the results from both 2D and 3D models and tried to define the best $2 \mathrm{D}$ representation of the reinforced material, in terms of geometric dimensions, which would be capable of displaying the same critical values as in 3D. In accordance with their conclusions, the width $2 e$ of the composite reinforcements in our 2D model is chosen in such a way that their second moment-to-area ratio is equal to the one of the real cylindrical reinforcements in the 3D material. The same critical loading (under through-thickness compression) is thus obtained in both 2D and 3D configurations in the absence of foam, since the buckling load of a beam-like structure is governed by its bending rigidity. Moreover, the width $(2 H)$ of the foam blocks separating the successive reinforcements is defined so as to maintain the same volume fraction of reinforcements between the $2 \mathrm{D}$ and $3 \mathrm{D}$ configurations. This particular choice has proved to give satisfactory results for the buckling problem of a reinforced sandwich under through-thickness compression. Eventually, the thicknesses of the core layer $(2 L)$ and of the two identical faces $(2 t)$ are the real $3 \mathrm{D}$ ones measured experimentally.

The problem in hand is represented in Figure 5. It consists in a complete sandwich column of total length $l$ containing $N$ reinforcements (that is to say $N$ consecutive cells). The column is clamped at the left end and guided at the right end. These boundary conditions are particularized so as to clarify expectations without limiting the general scope of the subsequent developments.

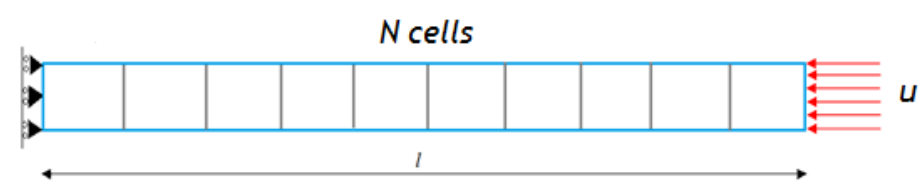

Figure 5: Reinforced sandwich column ( $N$ cells) under axial compression

The objective of the paper is to find an efficient method for the evaluation of the successive critical values and corresponding buckling modes of such a reinforced sandwich beam. For this purpose, a unit cell model is defined, comprising a foam block, two skins and two half-reinforcements, see Figure 6. It will be used in the sequel in order to derive efficiently the critical values of the total beam, by means of the Bloch wave theory.

\subsection{Bloch wave theory}

The Bloch wave theory, initially introduced for the analysis of wave functions for particles (such as electrons) in a periodically-repeating environment (such as crystals), has then long been utilized in acoustics 


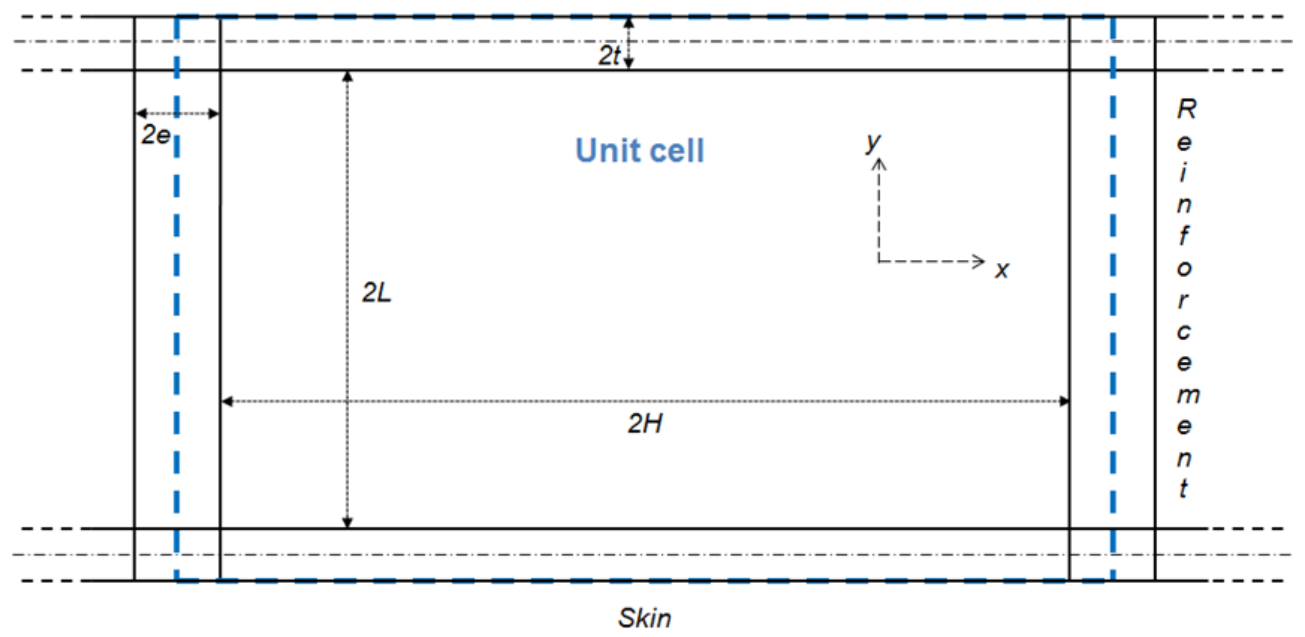

Figure 6: Unit cell model for the numerical modeling

and more specifically for the description of wave propagation in periodic porous or composite media. For instance, Boutin et al. 14 analyzed the large scale modulations of high frequency waves in periodic elastic structures in the context of the Bloch wave theory. As the buckling response of periodic composites often reveals critical local modes with large wave lengths compared to the typical length of a unit cell, the Bloch wave theory is utterly appropriate for solving such stability problems, but nonetheless it has been far less operated in this context. Let us mention a few papers on buckling among the rare involving a Bloch wave analysis. Bertoldi et al. 15] performed Bloch wave calculations so as to analyze the pattern transformations of infinite periodic elastomeric cellular structures under compressive loading. More recently, Liu and Bertoldi [16] studied the wrinkling behavior of bilayer structures made of a thin film bonded on a compliant substrate under compression. In this work, the authors analyzed not only the buckling but also the post-buckling response and revealed the presence of secondary critical modes that were also identified by means of the Bloch wave theory. Gong et al. [17] studied the buckling and post-buckling behavior of Kelvin cell foams under compressive loads by also using the Bloch wave theory. The foams were considered to be periodic and the ligaments were modeled as shear deformable beams. The post-buckling response was found to be either stable or unstable (involving a localization phenomenon), depending on whether the mode is local or global (with long wave lengths). López Jiménez et Triantafyllidis [5] analyzed the buckling behavior of various honeycomb structures (to be used as the core layer in sandwich structures) under out-of-plane compression and/or transverse shear, relying on the Bloch wave representation theorem. Wang and Abdalla [10] investigated the buckling behavior of grid-stiffened composite panels, including the particular case of composite stiffened cylindrical shells under axial compression. The local critical modes, involving both the skins and stiffeners, were specifically obtained through a Bloch wave analysis (see also [18] where curved stiffeners are designed optimally against buckling). The case of stiffened or corrugated 
axially loaded cylindrical shells was also considered by Ning and Pellegrino [19]. A modified Bloch wave method was developed, so as to be applied to rotationally periodic structures, and it showed to be able to capture both global and local modes with a very good accuracy. The main interest of the use of the Bloch wave approach in all previous investigations is the significant reduction of computational time.

In this paper, a Bloch wave analysis is also achieved in order to obtain first in an efficient way (at a low computational cost) all the global and local buckling modes of a reinforced sandwich column under longitudinal compression. A specific numerical approach, where all the calculations are performed on a unit cell model, is identified and implemented in the commercial finite element software Abaqus. The successive steps of the procedure are presented in the sequel.

\subsection{Numerical procedure}

The sandwich column in hand is periodically reinforced along the longitudinal direction, so that one can define and rely on the primitive unit cell illustrated in Figure 6. The general idea is to derive the critical loadings and corresponding buckling modes of the column by simply performing linearized buckling analyses on the previous unit cell, through the use of appropriate Bloch-periodic conditions. Since such conditions cannot be applied on the left-hand side and right-hand side of the unit cell while seeking the critical force or displacement (which needs to be applied to the same edges) resulting in buckling, an indirect method is retained, based on the computation of the natural frequencies of the pre-stressed unit cell. The general procedure may be divided into the four following steps.

\subsubsection{Compressive strain}

First of all, the unit cell is pre-stressed by introducing a compressive strain in the longitudinal direction. The simplest way to get such a quasi-uniform strain state in the unit cell is to prescribe the longitudinal displacement to zero on the left-hand side of the cell and to enforce a non-zero compressive displacement $\bar{u}$ on the right-hand side, see Figure 7(a). The average pre-strain in the longitudinal direction may then be defined by:

$$
\varepsilon_{x x}=\frac{u_{C D}-u_{A B}}{2 H}=\frac{\bar{u}}{2 H}
$$

where $[A B]$ and $[C D]$ are the left and right edges of the cell, respectively, see Figure 7(a). In Equation (1) and in all the subsequent analysis, the width of the reinforcements is neglected compared to the width of the foam blocks $(e \ll H)$, so that the total width of the unit cell is approximated by $2 H$. Similarly, the thickness of the skins will further be neglected compared to the one of the foam blocks $(t \ll L)$, for simplicity purposes.

Finally, it should be mentioned that, during this step, the vertical displacement of an arbitrary point has to be set to zero, in order to prevent from a rigid mode in the vertical direction. 


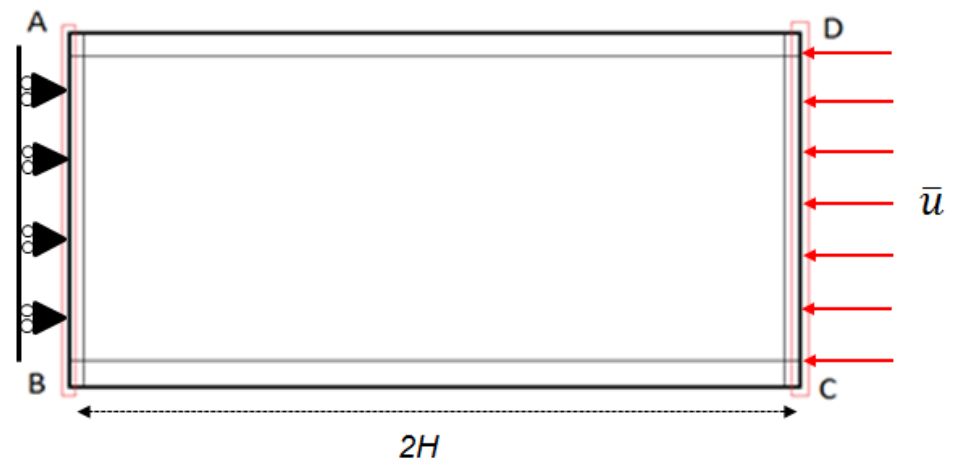

(a) Enforced compressive displacement

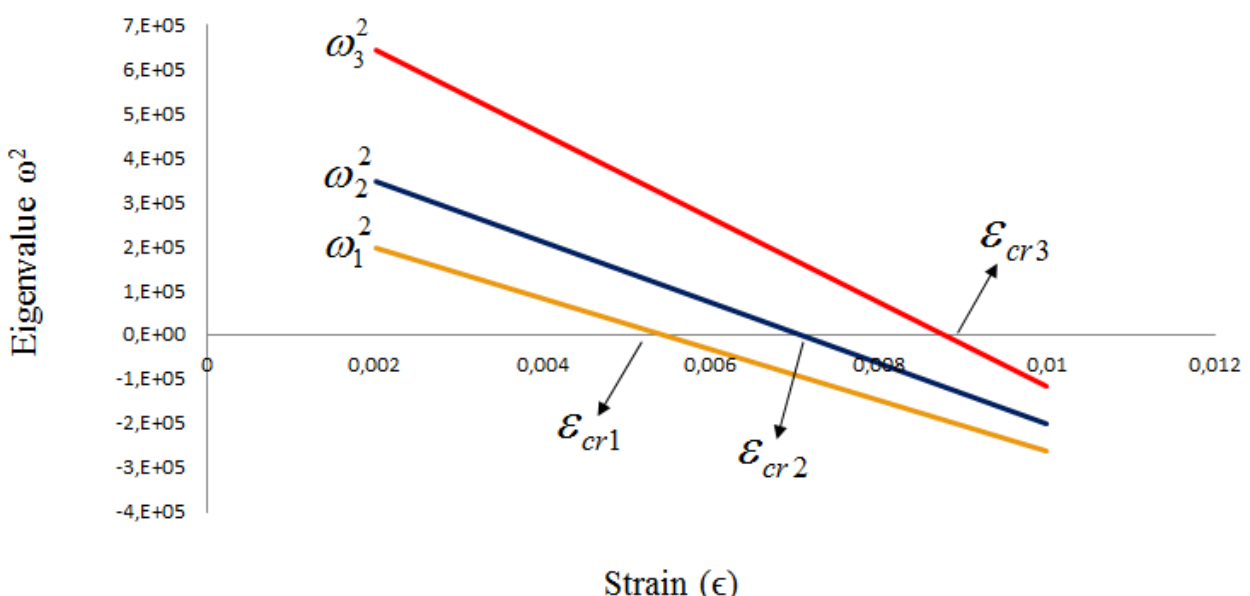

(b) Linear extrapolation of the eigenvalues

Figure 7: General procedure for the evaluation of the critical strains on a unit cell

\subsubsection{Natural frequencies}

A modal analysis is then performed on the pre-stressed unit cell, namely the following eigenvalue problem is solved:

$$
\left(\left[K_{T}\right]-\omega^{2}[M]\right)\{X\}=\{0\}
$$

In Equation (2), $[M]$ is the mass matrix of the unit cell model and $\left[K_{T}\right]$ its tangent stiffness matrix, depending on the pre-stress introduced by the enforced displacement in the previous step (the small amplitude vibrations of the unit cell are superimposed on the current state of deformation). In practice, a linearized frequency analysis is carried out and allows one to derive the sought natural frequencies $\omega$ and corresponding vibration modes $\{X\}$.

The Bloch wave theory comes into play here, throughout the choice of boundary conditions. The hori- 
zontal and vertical components of the eigenmodes (namely the modal displacements $U$ and $V$, respectively) are assumed to meet the so-called Floquet-Bloch periodic conditions. In such circumstances, it is possible to capture the probable change in periodicity between the geometric and material configuration and the modal solution. Since periodicity occurs here in only one direction, these conditions may simply write in the following form [16]:

$$
\begin{aligned}
& U_{C D}=U_{A B} \quad \exp (2 i k H) \\
& V_{C D}=V_{A B} \exp (2 i k H)
\end{aligned}
$$

involving a single wave number $k$.

Equations (3) are intrinsically complex ( $i$ is the imaginary number such that $i^{2}=-1$ ). A commercial software such as Abaqus is not able to handle such complex-valued relations, so that one has to split the equations into their real and imaginary parts. In practice, two identical unit cell models are used and the two real and imaginary problems are solved in parallel, as explained in Åberg and Gudmunson [20] when dealing with the computation of dispersion relations in materials with periodic microstructures. These two problems can be joined together in the following extended equation system:

$$
\left(\left[\begin{array}{cc}
K_{T} & 0 \\
0 & K_{T}
\end{array}\right]-\omega^{2}\left[\begin{array}{cc}
M & 0 \\
0 & M
\end{array}\right]\right)\left\{\begin{array}{l}
X^{R e} \\
X^{I m}
\end{array}\right\}=\left\{\begin{array}{l}
0 \\
0
\end{array}\right\}
$$

where $K_{T}$ and $M$ are the previous stiffness and mass matrices, and $X^{R e}$ and $X^{I m}$ stand for the real and imaginary parts of the modal displacements, respectively. The equation system (4) is uncoupled, but the real and imaginary boundary conditions are coupled to each other in the following way:

$$
\begin{aligned}
& U_{C D}^{R e}=U_{A B}^{R e} \cos (2 k H)-U_{A B}^{I m} \sin (2 k H) \\
& U_{C D}^{I m}=U_{A B}^{I m} \cos (2 k H)+U_{A B}^{R e} \sin (2 k H) \\
& V_{C D}^{R e}=V_{A B}^{R e} \cos (2 k H)-V_{A B}^{I m} \sin (2 k H) \\
& V_{C D}^{I m}=V_{A B}^{I m} \cos (2 k H)+V_{A B}^{R e} \sin (2 k H)
\end{aligned}
$$

In order to account for these coupled boundary conditions, a specific Fortran program is developed in the framework of Abaqus software. Moreover, the (linear) relationships above between degrees of freedom of nodes belonging to the left and right edges, respectively, are established using multi-point constraints (which is an available feature in Abaqus). Lastly, solving Equation (4) with boundary conditions (5) leads to coupled real and imaginary modes associated to each eigenvalue $\omega$, as depicted in Figure 8 in the periodic case $(k=0)$.

In most preceding stability analyses involving Bloch-periodic conditions, the structure is considered as of infinite length and calculations should be made for a large number of wave numbers in the following interval: $k \in[0, \pi / 2 H]$ (for a unit cell of length $2 H$ ). For each wave number considered in this interval, a critical strain is obtained (as explained below), and a critical wave number $k_{c r}$ can be identified as being 


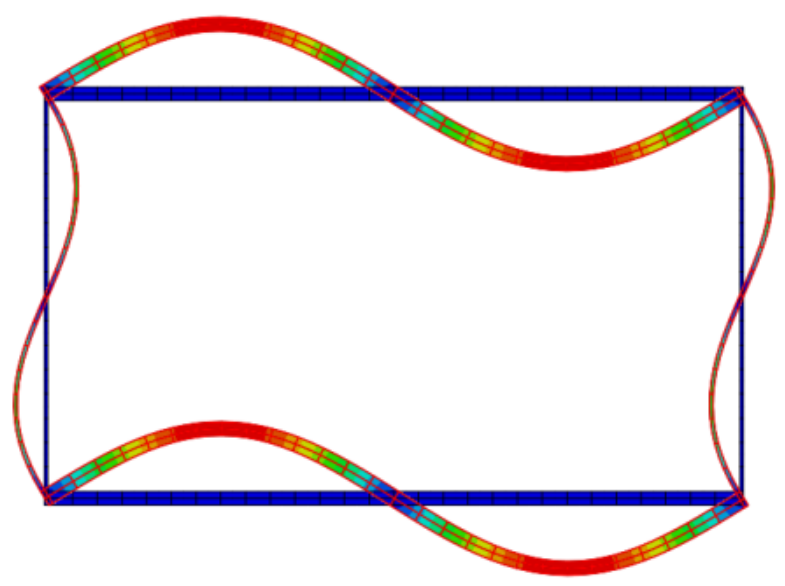

Figure 8: Periodic mode obtained with a unit cell model

the one associated to the smallest critical strain. A critical length $l_{c r}=2 \pi / k_{c r}$ can also be defined which corresponds to the periodic wave length of the first buckling pattern of the infinite structure. Owing to the finite size of the sandwich column here, only a finite number of values of $k$ needs to be investigated. Following the idea of Ning and Pellegrino 19 for rotationally periodic structures, it can be shown that the $N+1$ values $k=\frac{n \pi}{2 N H} \quad(n=0,1,2, \ldots, N)$ allow one to retrieve all the modes achieved with the complete sandwich column of $N$ cells with clamped-guided boundary conditions (these conditions lead to a particular constraint on the wave length of the mode which makes other values of $k$ unsuitable).

\subsubsection{Critical strain}

As the enforced compressive strain increases in magnitude, the natural frequencies gradually decrease and each buckling critical strain obtained for a given wave number $k$ can be viewed as the compressive strain at which the natural frequency corresponding to the concerned mode vanishes. Indeed, when considering a null value of $\omega$, Equation (2) simplifies and becomes:

$$
\left[K_{T}\right]\{X\}=\{0\}
$$

in such a way that the applied compressive strain makes the tangent stiffness matrix singular, which indicates an underlying buckling phenomenon.

In practice, the squared frequency $\omega^{2}$ is proved to be almost proportional to the applied strain, so that the use of two arbitrary strains allow one to extrapolate the results and find the sought critical strains. The procedure is illustrated in Figure 7(b), where the first three critical strains corresponding to a given arbitrary value of $k$ are extrapolated by linear interpolation of the squared frequencies. 


\subsubsection{From the unit cell to the whole structure}

Finally, for each critical strain determined with the unit cell model, one can rebuild the corresponding complete buckling mode, starting from the real and imaginary unit cell vibration modes, in accordance with the retained boundary conditions for the full column (see Figure 9p. The reconstruction technique is based on the following Bloch relations between the real and imaginary parts of the buckling mode in a first unit cell and the corresponding (real) modal displacements (with components $U_{n}$ and $V_{n}$ ) in all the $N$ successive unit cells of the entire column, varying $n$ from 0 to $N-1$ :

$$
\begin{aligned}
& U_{n}=U^{R e} \cos (2 n k H)-U^{I m} \sin (2 n k H) \\
& V_{n}=V^{R e} \cos (2 n k H)-V^{I m} \sin (2 n k H)
\end{aligned}
$$

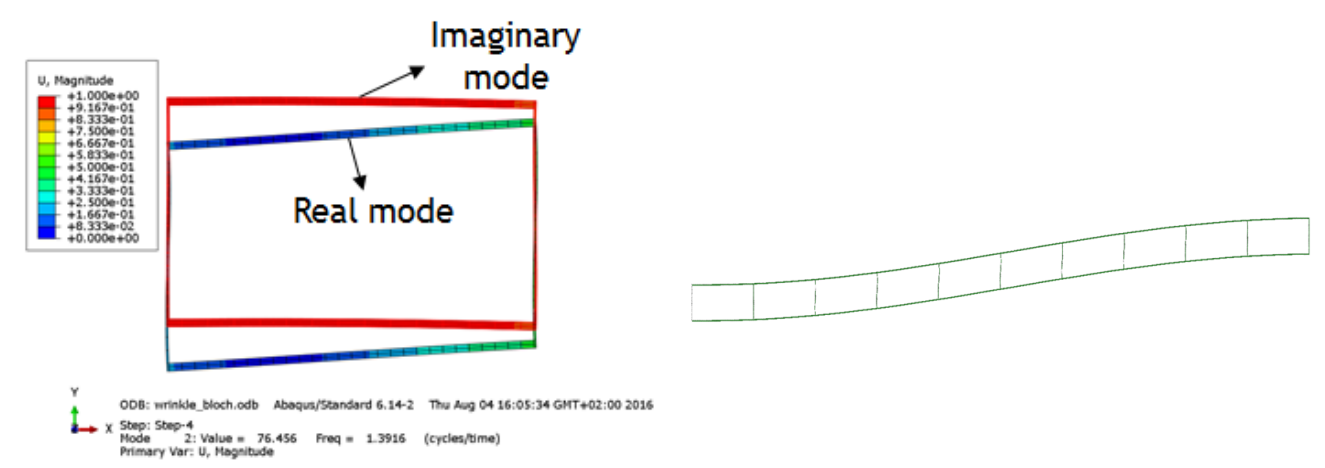

(a) Real and imaginary components of a unit cell (b) Corresponding rebuilt mode for the entire colmode umn

Figure 9: From the unit cell modes to the buckling behavior of the complete column

Let us finally mention that, for efficiency purposes, a Python program has been developed in Abaqus environment so as to automate the global (repetitive) procedure above.

\section{Analytical modeling}

Contrary to the general case of a sandwich column with many reinforcements, the unit cell problem is likely to be solved analytically, due to its simple configuration and the small number of components brought into play. This section is thus devoted to find an exact analytical solution for the buckling problem of a reinforced sandwich column under axial compression, still using a unit cell model and the Bloch wave representation of the critical modes. Although the numerical procedure above was presented in the general context of a reinforced sandwich including a foam core material, the analytical solution will be developed considering that the influence of this core material on the buckling behavior of the column is negligible compared to that of the skins and reinforcements, for simplicity purposes. This paper focuses on the use of 
the Bloch wave theory for the buckling analysis of reinforced columns, and it has been shown numerically (using the previous numerical tool) that, even if the core modulus may modify the critical values, it does not change at all the typical behavior which will be further described, mainly due to the coupling effect between the skins and the reinforcements. As a consequence, the core material will also be removed in the subsequent validation and analysis of the results.

In the end, the new unit cell model of Figure 10 is considered for conveniency purposes, which is equivalent to the one displayed in Figure 6. It consists of one reinforcement and two skin segments with the same geometric and material properties as before.

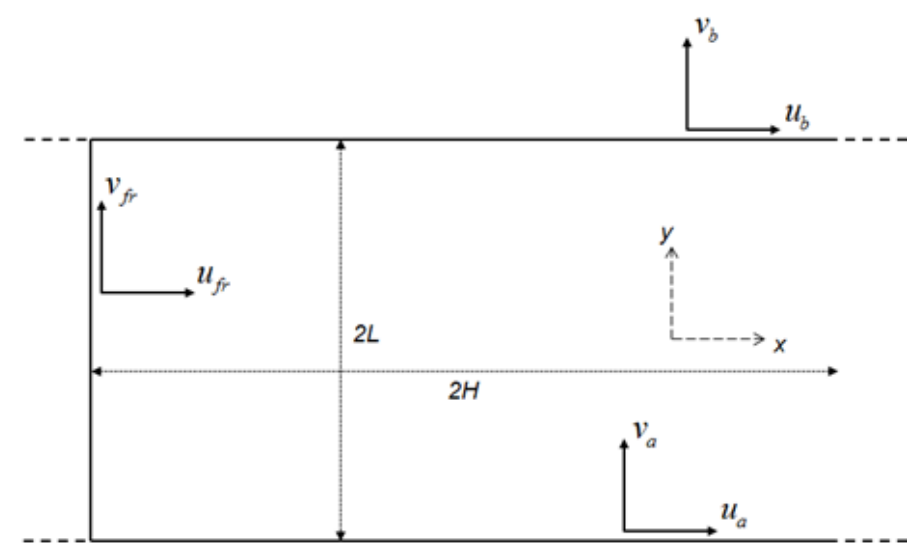

Figure 10: Unit cell model for the analytical modeling

\subsection{Theoretical formulation}

The model used here to investigate the elastic buckling behavior of a reinforced sandwich column under axial compression is very similar to the one already developed in [8] and [4] for the buckling analysis of reinforced sandwiches under through-thickness compression and classical sandwiches (without reinforcements) under axial compression. The main difficulty here comes from the fact that both skins and reinforcements may buckle simultaneously, whereas only the reinforcements or the skins were involved in the previous studies, respectively in [8] and [4].

The critical displacements (or strains) and the associated bifurcation modes are derived from a 3D framework: the theory is developed using a total Lagrangian formulation where the different components of the model are initially seen as 3D bodies. This method has already been applied successfully to the previous cases. The facings and reinforcement are then assumed to behave like Euler-Bernoulli beams, with a linear elastic constitutive law. Due to these kinematic hypotheses, only their Young's modulus ( $E_{s}$ and $E_{f r}$, respectively) will be involved subsequently. 
The critical loading $\lambda_{c r}$ and the bifurcation mode $\mathbf{X}$ of a 3D body can be generally obtained by solving the following bifurcation equation:

$$
\forall \delta \mathbf{U}, \quad \int_{\Omega} \nabla^{T} \delta \mathbf{U}: \mathbf{K}\left(\lambda_{c r}\right): \nabla \mathbf{X} d \Omega=0
$$

where $\Omega$ is the volume of the $3 \mathrm{D}$ body in the reference configuration and $\delta \mathbf{U}$ stands for the variation of the displacement field in the body.

The fourth-order nominal tangent elastic tensor $\mathbf{K}$ can be written as follows:

$$
\mathbf{K}=\frac{\partial \boldsymbol{\Pi}}{\partial \mathbf{F}}=\mathbf{F} \cdot \frac{\partial \boldsymbol{\Sigma}}{\partial \mathbf{E}} \cdot \mathbf{F}^{T}+(\mathbb{I} \cdot \boldsymbol{\Sigma})^{T}=\mathbf{F} \cdot \mathbf{D} \cdot \mathbf{F}^{T}+(\mathbb{I} \cdot \boldsymbol{\Sigma})^{T}
$$

In the above equation, $\mathbf{E}$ denotes the Green strain tensor and $\boldsymbol{\Sigma}$ the second Kirchhoff stress tensor (symmetric). $\mathbf{F}$ is the deformation gradient and $\mathbf{\Pi}=\mathbf{F} . \boldsymbol{\Sigma}$ the first Kirchhoff stress tensor (non-symmetric). $\mathbb{I}$ represents the fourth-order unit tensor $\left(I_{i j k l}=\delta_{i l} \delta_{k j}\right)$ and the superscript ${ }^{T}$ the transposition of a secondorder tensor and the major transposition of a fourth-order tensor $\left(\left(\mathbf{A}^{T}\right)_{i j k l}=A_{k l i j}\right)$, respectively. The fourth-order material tangent elastic tensor $\mathbf{D}$ of an isotropic material can be defined by its components in an orthonormal basis $D_{i j k l}=\Lambda \delta_{i j} \delta_{k l}+\mu\left(\delta_{i k} \delta_{j l}+\delta_{i l} \delta_{k j}\right)$, where $\delta_{i j}$ is the Kronecker symbol, and $\Lambda$ and $\mu$ are the Lamé constants. Use is also made of the Young's modulus $E$, the Poisson's ratio $\nu$ and the shear modulus $G$ related to $\Lambda$ and $\mu$ by the standard relations $\Lambda=\frac{E \nu}{(1+\nu)(1-2 \nu)}$ and $\mu=G=\frac{E}{2(1+\nu)}$.

We shall now derive more explicit expressions of the above tensors by exploiting the uniaxial stress state in the body. More precisely, while the uniform compressive displacement applied on the unit cell obviously leads to compressive stresses in the facings, the reinforcement will be supposed to be free of initial stresses at the onset of buckling.

On one hand, the skins are thus subjected to a nominal axial compressive stress $\Pi_{x x}=-P<0$ in their longitudinal direction, so that the first Kirchhoff stress tensor $\boldsymbol{\Pi}$ is expressed in the orthonormal basis $\left(\mathbf{e}_{x}, \mathbf{e}_{y}, \mathbf{e}_{z}\right)$ as:

$$
\boldsymbol{\Pi}=-P \mathbf{e}_{x} \otimes \mathbf{e}_{x}=\left[\begin{array}{ccc}
-P & 0 & 0 \\
0 & 0 & 0 \\
0 & 0 & 0
\end{array}\right] \quad(P>0)
$$

Let us make the assumption that the pre-critical deformations are small, which is usually satisfied in practice:

$$
\|\nabla \mathbf{U}\| \ll 1
$$

Thus, the stress tensor $\boldsymbol{\Sigma}$ writes:

$$
\boldsymbol{\Sigma}=\mathbf{F}^{-1} . \mathbf{\Pi} \approx \boldsymbol{\Pi}
$$

The nominal tangent elastic tensor in Equation 9 becomes then:

$$
\mathbf{K} \approx \frac{\partial \boldsymbol{\Sigma}}{\partial \mathbf{E}}+(\mathbb{I} . \boldsymbol{\Sigma})^{T}=\mathbf{D}-P \mathbf{e}_{i} \otimes \mathbf{e}_{x} \otimes \mathbf{e}_{x} \otimes \mathbf{e}_{i}
$$


which is independent of the spatial coordinates (the implicit summation convention on repeated indices is used with $i=x, y, z)$.

Furthermore, when dealing with 1D models like beams, ad hoc assumptions are usually added in order to enforce some specific stress state in the body. Namely, the transverse normal material stresses are assumed to be zero: $\Sigma_{y y}=\Sigma_{z z}=0$. Taking into account these assumptions leads one to replace tensor $\mathbf{D}$ with the reduced tensor $\mathbf{C}$ defined as:

$$
\begin{aligned}
C_{i j k l}=D_{i j k l}+\frac{D_{i j y y}\left(D_{y y z z} D_{z z k l}-D_{z z z z} D_{y y k l}\right)+D_{i j z z}\left(D_{z z y y} D_{y y k l}-D_{y y y y} D_{z z k l}\right)}{D_{y y y y} D_{z z z z z}-D_{y y z z} D_{z z y y}} \\
(i, j) \neq(y, y),(z, z) \quad(k, l) \neq(y, y),(z, z)
\end{aligned}
$$

It can be readily checked that tensor $\mathbf{C}$ has the major and both minor symmetries. In the sequel, we only need the following reduced moduli (and their equivalents obtained by major or minor symmetries):

$$
C_{x x x x}=E \quad C_{x y x y}=C_{x z x z}=C_{y z y z}=G
$$

where only $E=E_{s}$ will explicitly appear in the final bifurcation equation.

On the other hand, as the existing initial stresses in the reinforcement at the critical point are neglected, the nominal tangent elastic tensor in this case simply writes:

$$
\mathbf{K} \approx \frac{\partial \boldsymbol{\Sigma}}{\partial \mathbf{E}}=\mathbf{D}
$$

which may also be replaced by its reduced counterpart $\mathbf{C}$ (due to the kinematics, the Young's modulus $E_{f r}$ will be again the only parameter appearing in the final equations).

Eventually, the bifurcation equation (8) of the unit cell writes:

$$
\begin{gathered}
\forall \delta \mathbf{U}_{a}, \delta \mathbf{U}_{b}, \delta \mathbf{U}_{f r}, \quad \int_{\Omega_{a}} \nabla^{T} \delta \mathbf{U}_{a}:\left(\mathbf{C}^{s}-P_{c r} \mathbf{e}_{i} \otimes \mathbf{e}_{x} \otimes \mathbf{e}_{x} \otimes \mathbf{e}_{i}\right): \nabla \mathbf{X}_{a} d \Omega_{a} \\
+\int_{\Omega_{b}} \nabla^{T} \delta \mathbf{U}_{b}:\left(\mathbf{C}^{s}-P_{c r} \mathbf{e}_{i} \otimes \mathbf{e}_{x} \otimes \mathbf{e}_{x} \otimes \mathbf{e}_{i}\right): \nabla \mathbf{X}_{b} d \Omega_{b}+\int_{\Omega_{f r}} \nabla^{T} \delta \mathbf{U}_{f r}: \mathbf{C}^{f r}: \nabla \mathbf{X}_{f r} d \Omega_{f r}=0
\end{gathered}
$$

The compressive stress $(P>0)$, identical for the two skins, is related to the enforced strain $\varepsilon>0$ (which will act as the bifurcation parameter) by the following relation:

$$
P=E_{s} \varepsilon
$$

and $\mathbf{U}_{a}, \mathbf{U}_{b}, \mathbf{U}_{f r}, \mathbf{X}_{a}, \mathbf{X}_{b}$ and $\mathbf{X}_{f r}$ represent the displacement field and bifurcation mode components, respectively, relative to the bottom and top skins (with indices $\bullet_{a}$ and $\bullet_{b}$, respectively) and the reinforcement (with indice $\bullet_{f r}$ ).

Let us now consider the bending problem of the skins and the reinforcement in the $x y$-plane. The Euler-Bernoulli beam theory is employed, as transverse shear effects may be negligible in practice (due to the low thickness-to-length ratio of all the components). The Euler-Bernoulli kinematics is defined by two scalar displacement fields, respectively the axial and transverse displacements of the centroid axis of the 
beam. When the skins buckle from the straight position (the fundamental solution) to a bent shape, the expressions for the bifurcation modes $\mathbf{X}_{i}$ and the displacement variations $\delta \mathbf{U}_{i}$ (with $i=a, b$ depending on the skin considered) are both chosen accordingly as follows:

$$
\mathbf{X}_{i}=\mid \begin{array}{ll}
U_{i}-y V_{i},{ }_{x} & \delta \mathbf{U}_{i}=\mid \begin{array}{l}
\delta u_{i}-y \delta v_{i}, x_{x} \\
V_{i} \\
0
\end{array} \\
0
\end{array}
$$

The bifurcation mode $\mathbf{X}_{f r}$ and the displacement variation $\delta \mathbf{U}_{f r}$ in the reinforcement are defined with the same kinematics as:

$$
\mathbf{X}_{f r}=\mid \begin{array}{l|l}
U_{f r} & \delta \mathbf{U}_{f r}= \\
V_{f r}-x U_{f r}, y & \delta u_{f r} \\
0 & \delta v_{f r}-x \delta u_{f r}, y \\
0
\end{array}
$$

The global bifurcation equation then writes:

$$
\begin{gathered}
\forall \delta u_{a}, \delta v_{a}, \delta u_{b}, \delta v_{b}, \delta u_{f r}, \delta v_{f r}, \quad \int_{\Omega_{a}}\left[E_{s}\left(U_{a, x}-y V_{a},_{x x}\right)\left(\delta u_{a, x}-y \delta v_{a, x x}\right)\right. \\
-E_{s} \varepsilon_{c r}\left(U_{a, x}-y V_{a}, x x\right)\left(\delta u_{a}-y \delta v_{a}, x x-E_{s} \varepsilon_{c r} V_{a},_{x} \delta v_{a, x}\right] d \Omega_{a} \\
\quad+\int_{\Omega_{b}}\left[E_{s}\left(U_{b, x}-y V_{b, x x}\right)\left(\delta u_{b, x}-y \delta v_{b}, x x\right)\right. \\
\left.-E_{s} \varepsilon_{c r}\left(U_{b, x}-y V_{b, x x}\right)\left(\delta u_{b}, y \delta v_{b, x x}\right)-E_{s} \varepsilon_{c r} V_{b, x} \delta v_{b, x}\right] d \Omega_{b} \\
+\int_{\Omega_{f r}}\left[E_{f r}\left(V_{f r, y}-x U_{f r, y y}\right)\left(\delta v_{f r, y}-x \delta u_{f r}, y y\right)\right] d \Omega_{f r}=0
\end{gathered}
$$

where $y$ (respectively $x$ ) stands for the $y$-coordinate (respectively $x$-coordinate) of a current point relative to the centroid axis of the corresponding skin (respectively reinforcement).

First, integrating over the cross-sections of the beams, then integrating by parts with respect to $x$ or $y$, and eliminating negligible higher-order terms (presupposing that $\varepsilon_{c r} \ll 1$ ) yields six local differential equations for the components $U_{a}, V_{a}, U_{b}, V_{b}, U_{f r}$ and $V_{f r}$ of the eigenmode:

$$
\begin{aligned}
& U_{a, x x}=0 \\
& \frac{1}{3} h_{s}^{2} V_{a},{ }_{x x x x}+\varepsilon_{c r} V_{a}, x x=0 \\
& U_{b}, x x=0 \\
& \frac{1}{3} h_{s}^{2} V_{b}, x x x x+\varepsilon_{c r} V_{b}, x x=0 \\
& U_{f r, y y y y}=0 \\
& V_{f r, y y}=0
\end{aligned}
$$

At this stage, one has to specify the boundary conditions in order to solve the previous system. First, connecting conditions for the displacement fields (bifurcation mode) must be satisfied at the intersection between the reinforcement and the two facings. Due to the entanglement of the fibres, the two ends of the 
reinforcement can be considered as clamped in the skins, which leads to the following relationships:

$$
\begin{aligned}
& U_{a}(-H)=U_{f r}(-L) \\
& V_{a}(-H)=V_{f r}(-L) \\
& V_{a},,_{x}(-H)=-U_{f r}, y \\
& U_{b}(-H)=U_{f r}(L) \\
& V_{b}(-H)=V_{f r}(L) \\
& V_{b, x}(-H)=-U_{f r, y}(L)
\end{aligned}
$$

Calculations are performed on a unit cell, where Bloch-periodic boundary conditions are used (instead of periodic ones as in [8]), so as to deal with any global or local mode whose period does not necessarily coincide with the size of the unit cell. Taking into account these Bloch-periodic conditions between the two ends of each face leads to the following kinematical constraints:

$$
\begin{aligned}
& U_{a}(H)=U_{a}(-H) \exp (2 i k H) \\
& V_{a}(H)=V_{a}(-H) \exp (2 i k H) \\
& V_{a},,_{x}(H)=V_{a},,_{x}(-H) \exp (2 i k H) \\
& U_{b}(H)=U_{b}(-H) \exp (2 i k H) \\
& V_{b}(H)=V_{b}(-H) \exp (2 i k H) \\
& V_{b},{ }_{x}(H)=V_{b},{ }_{x}(-H) \exp (2 i k H)
\end{aligned}
$$

After integration by parts, the remaining stress boundary conditions are obtained together with the differential equations $(22)$ :

$$
\begin{aligned}
& \frac{2}{3} E_{f r} e^{3} U_{f r}, y y y(-L)-2 E_{s} t U_{a, x}(-H)+2 E_{s} t U_{a,{ }_{x}}(H) \exp (-2 i k H)=0 \\
& -2 E_{f r} e V_{f r}, y(-L)+\frac{2}{3} E_{s} t^{3} V_{a}, x x x(-H)+2 E_{s} t \varepsilon_{c r} V_{a}, x(-H)-\frac{2}{3} E_{s} t^{3} V_{a}, x x x(H) \exp (-2 i k H) \\
& -2 E_{s} t \varepsilon_{c r} V_{a, x}(H) \exp (-2 i k H)=0 \\
& -\frac{2}{3} E_{f r} e^{3} U_{f r, y y}(-L)+\frac{2}{3} E_{s} t^{3} V_{a},_{x x}(-H)-\frac{2}{3} E_{s} t^{3} V_{a},_{x x}(H) \exp (-2 i k H)=0 \\
& -\frac{2}{3} E_{f r} e^{3} U_{f r, y y y}(L)-2 E_{s} t U_{b, x}(-H)+2 E_{s} t U_{b, x}(H) \exp (-2 i k H)=0 \\
& 2 E_{f r} e V_{f r, y}(L)+\frac{2}{3} E_{s} t^{3} V_{b}, x x x(-H)+2 E_{s} t \varepsilon_{c r} V_{b, x}(-H)-\frac{2}{3} E_{s} t^{3} V_{b}, x x x(H) \exp (-2 i k H) \\
& -2 E_{s} t \varepsilon_{c r} V_{b, x}(H) \exp (-2 i k H)=0 \\
& \frac{2}{3} E_{f r} e^{3} U_{f r, y y}(L)+\frac{2}{3} E_{s} t^{3} V_{b}, x x(-H)-\frac{2}{3} E_{s} t^{3} V_{b},_{x x}(H) \exp (-2 i k H)=0
\end{aligned}
$$




\subsection{Solution procedure}

The previous differential equation system (22) is totally uncoupled and gives rise to the general following solutions:

$$
\begin{aligned}
& U_{a}(x)=a_{0}+a_{1} x \\
& U_{b}(x)=b_{0}+b_{1} x \\
& V_{f r}(y)=c_{0}+c_{1} y \\
& V_{a}(x)=\alpha_{0}+\alpha_{1} x+\alpha_{2} \cos (p x)+\alpha_{3} \sin (p x) \\
& V_{b}(x)=\beta_{0}+\beta_{1} x+\beta_{2} \cos (p x)+\beta_{3} \sin (p x) \\
& U_{f r}(y)=\gamma_{0}+\gamma_{1} y+\gamma_{2} y^{2}+\gamma_{3} y^{3}
\end{aligned}
$$

with $p=\frac{\sqrt{3 \varepsilon_{c r}}}{t}$ and involving a set of 18 constant unknowns which will be further collected in a single vector:

$$
\{B\}^{T}=\left\langle a_{0}, a_{1}, b_{0}, b_{1}, c_{0}, c_{1}, \alpha_{0}, \alpha_{1}, \alpha_{2}, \alpha_{3}, \beta_{0}, \beta_{1}, \beta_{2}, \beta_{3}, \gamma_{0}, \gamma_{1}, \gamma_{2}, \gamma_{3}\right\rangle
$$

The verification of the boundary conditions 23 25 all at once may be written in the form of a linear equation system as follows:

$$
[A]\{B\}=\{0\}
$$

where $[A]$ has been reported in Appendix A for clarity purposes.

Equation (28) has a non-zero solution for vector $\{B\}$ once matrix $[A]$ is singular. The critical strains are thus obtained by solving:

$$
\operatorname{det}([A])=0
$$

Equation 29 is a complex-valued transcendent equation which is numerically solved for each successive value of the wave number $k$. Here, no separation between real and imaginary parts are required, since Equation 29 can be directly solved in the plane of complex numbers. Nevertheless, all the solutions of Equation 29 are found to be real critical strains, whatever the wave number $k$. In practice, for each critical strain $\varepsilon_{c r}$, the corresponding vector $\{B\}$ allows one to identify the modal displacement field, which can be split into real and imaginary parts so as to rebuild the related buckling mode of the complete sandwich column, using the same expressions $(7)$ as before.

\section{Validation and analysis of the results}

\subsection{Numerical finite element models}

For validation purposes, an illustrative example is presented in this section. The numerical values of the geometric and material parameters are listed in Table 1 (the foam core is not included in the model).

The numerical procedure presented above is applied on the finite element (unit cell) model depicted in Figure 11. The skins and reinforcement are represented by a $2 \mathrm{D}$ continuous solid satisfying the plane 


\begin{tabular}{|c|c|c|c|c|c|c|c|}
\hline$E_{s}(M P a)$ & $\nu_{s}$ & $E_{f r}(M P a)$ & $\nu_{f r}$ & $H(m m)$ & $L(m m)$ & $e(m m)$ & $t(m m)$ \\
\hline 9000 & 0.3 & 6834 & 0.39 & 13.95 & 8.075 & 0.1395 & 0.275 \\
\hline
\end{tabular}

Table 1: Geometric and material parameters for the unit cell model

stress hypothesis. Two-dimensional 8-noded quadrangular finite elements are thus employed, with quadratic shape functions and reduced integration. Only two elements are proved to be sufficient in the thickness of the skins and reinforcements (one for each half of the reinforcement), whereas the number of elements in the longitudinal direction of each component is obtained through a convergence analysis.

Remark. The use of 2D solid elements (instead of beam structural ones which would have been more efficient) is here more convenient for the definition of the two half-reinforcements and the application of the Bloch conditions, and it will make it easier to generalize to the case of a unit cell with the foam core included.

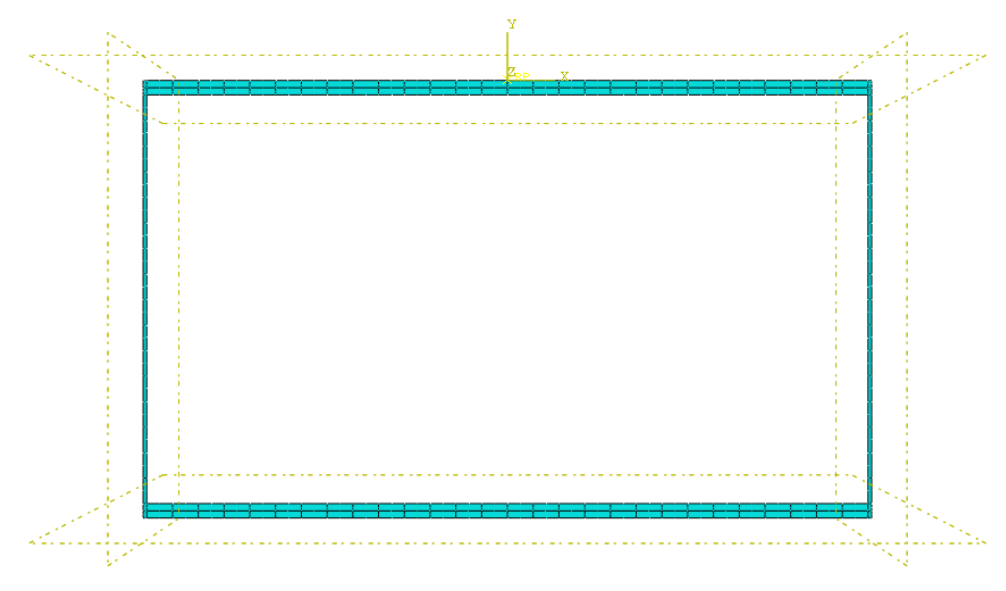

Figure 11: FE mesh used for the unit cell model

The finite element model of the complete sandwich column is derived from the previous one by juxtaposing the required number of cells side by side, while retaining the same mesh for each cell. An arbitrary number of $N=10$ cells is retained here. The left end section of the sandwich column is fixed horizontally whereas the right end section is submitted to a uniform horizontal displacement so as to trigger the buckling phenomenon (the vertical displacement of one arbitrary node is fixed in order to prevent the structure from rigid modes). Linearized buckling analyses are performed on this complete model, also using Abaqus software, so as to serve as reference results and validate the critical strains and modes provided by the Bloch wave theory on a unit cell. 


\subsection{Results and validation}

When considering the unit cell solutions, each critical strain corresponds to a double buckling mode: the two buckling modes corresponding to the same critical value are always the same, except that the roles are reversed between the real and imaginary components. For each value of $k=\frac{n \pi}{20 H} \quad(n=0,1,2, \ldots, 10)$, the first three (different) buckling modes are identified using the numerical and analytical unit cell models. It allows one to recover the first thirty modes of the complete sandwich column (the last one being double), which are found to be either symmetric or antisymmetric. Only four modes obtained with the numerical unit cell model are not observed in the complete column (they are thus not calculated with the analytical approach). These modes correspond to cases where the wave length is a multiple of the unit cell length and all the reinforcements (without exception) clearly deform (so that these modes can not comply to the applied boundary conditions on the complete column). Figure 12 displays these four modes that are obviously not obtainable when clamping both skins at the two ends of the column.

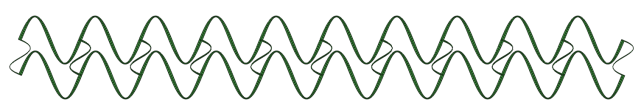

(a) $k=0-2^{\text {nd }}$ mode

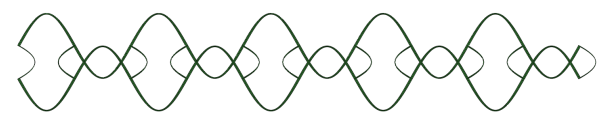

(c) $k=\frac{10 \pi}{20 H}-2^{n d}$ mode

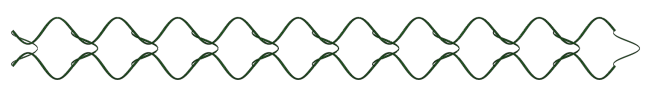

(b) $k=0-3^{r d}$ mode

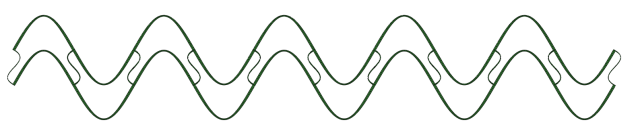

(d) $k=\frac{10 \pi}{20 H}-3^{r d}$ mode

Figure 12: Examples of rebuilt modes from unit cell calculations which are not obtained with the complete column

The associated critical displacements $\left(u_{c r}=\varepsilon_{c r} l\right)$ obtained with these three models are compared with each other and show a very good agreement, with a maximum relative error less than 1\% (see Table B.2 in

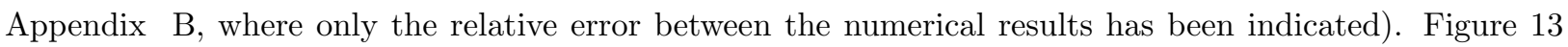
displays the evolution of these critical displacements with the mode number, which appear to be regularly distributed, and Figure 14 compares the buckling modes of the complete column obtained either directly with the complete model or after rebuilt from the unit cell results (only a selection of modes are presented, namely the ones with a wave length multiple of the unit cell dimension). The mode shapes are in total accordance between the two approaches. 


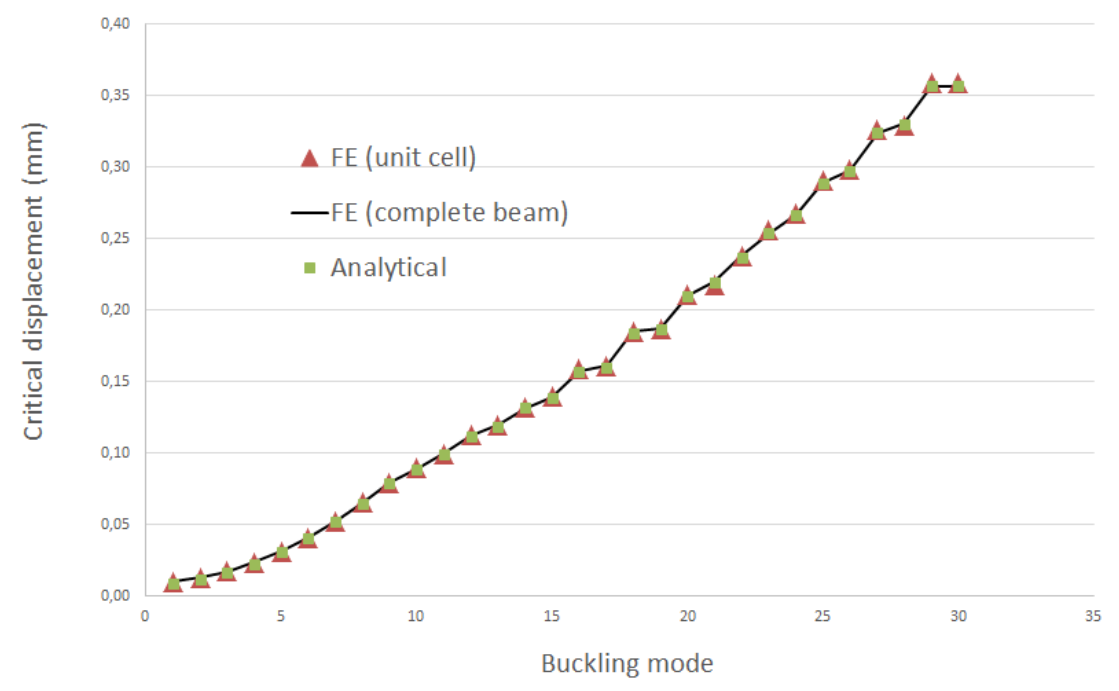

Figure 13: Comparison between the analytical and numerical critical displacements

\section{Conclusions}

The main objective of this paper is to give a first insight of the buckling behavior of sandwich structures reinforced thanks to the Napco ${ }^{\circledR}$ technology. This patented process transversally strengthens the foam core of a sandwich structure with fibre yarns taken from facings. In this study, we investigated the influence of such reinforcements on the buckling behavior of sandwich columns under axial compression. Thanks to the periodicity properties of the buckling modes obtained with classical linearized buckling analyses on the whole structures, an efficient numerical methodology was proposed, based on the use of a single unit cell. It can be separated into three successive steps, namely (i) the longitudinal compression of the unit cell (with two arbitrary strains), (ii) the calculation of natural frequencies and corresponding vibration modes and (iii) the extrapolation of the critical strains for each mode. As the wave length of the modes may be different from the unit cell length, the use of Bloch-periodic conditions are required instead of purely periodic ones. It gives rise to complex-valued expressions which result in a specific solution procedure with two real and imaginary almost identical problems, when requesting the use of a commercial finite element software. Simultaneously, analytical solutions were obtained in the simplified case where the foam core is removed. Using an Euler-Bernoulli beam formulation for both skins and reinforcements, the critical strains and modes were derived from a 3D bifurcation analysis of a unit cell similar to the one used in the numerical procedure.

2D finite element models were developed for the unit cell and, by extension, for the entire column. Unit cell analytical and numerical solutions were validated against full numerical computations performed on the sandwich column, in the particular case considered analytically. The critical strains (or displacements) 


\begin{tabular}{|c|c|c|c|}
\hline $\begin{array}{c}\text { Mode } \\
\text { number }\end{array}$ & $k$ & Unit cell & Complete beam \\
\hline 1 & $\frac{\pi}{20 H}$ & III & | \\
\hline 2 & $\frac{2 \pi}{20 H}$ & I & $M$ \\
\hline 4 & $\frac{4 \pi}{20 H}$ & & \\
\hline 5 & $\frac{5 \pi}{20 H}$ & & \\
\hline 10 & $\frac{10 \pi}{20 H}$ & & \\
\hline 19 & $\frac{5 \pi}{20 H}$ & & \\
\hline 20 & $\frac{5 \pi}{20 H}$ & N & \\
\hline 21 & $\frac{4 \pi}{20 H}$ & & \\
\hline 22 & $\frac{4 \pi}{20 H}$ & NANAN & s \\
\hline 25 & $\frac{2 \pi}{20 H}$ & Noxerswaris & \\
\hline 26 & $\frac{2 \pi}{20 H}$ & 公企企 & ANAan \\
\hline $29 / 30$ & 0 & manswan & nas \\
\hline
\end{tabular}

Figure 14: Comparison between representative buckling modes obtained with the complete beam model and corresponding modes rebuilt from the unit cell real and imaginary solutions

and the corresponding modes were shown to compare very well with each other. It means that both the analytical and numerical procedures defined on the unit cell allow one to estimate very efficiently and with a 
very good accuracy the critical values and modes of a given sandwich column, as soon as the wave numbers considered in the Bloch wave analysis are consistent with the boundary conditions imposed to the complete column.

Since the methodology developed here is general, the present results may be adapted to the case of reinforced sandwich columns including a foam core material (in the analytical case, it demands further calculation efforts, but it is straightforward in the numerical case) and eventually to the case of sandwich plates under biaxial compression(-tension), or other loading conditions. 
Appendix A. Global matrix for the verification of the boundary conditions

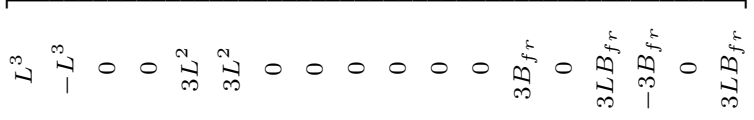

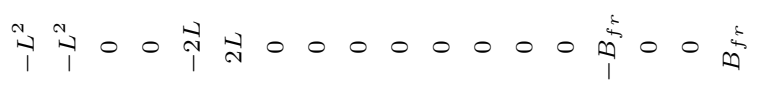

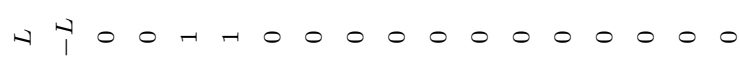

$$
\begin{aligned}
& \vec{\imath} \overrightarrow{1} 000000000000000
\end{aligned}
$$

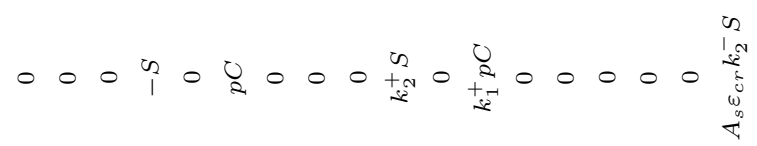

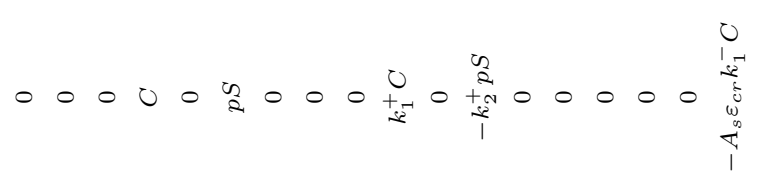

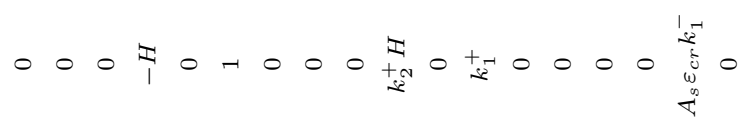

$$
\begin{aligned}
& 000-00000,700000000
\end{aligned}
$$

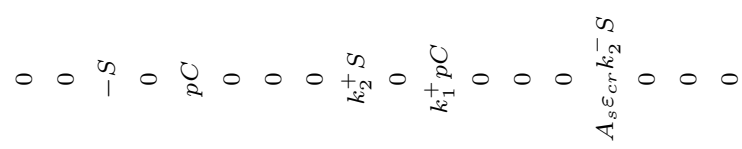

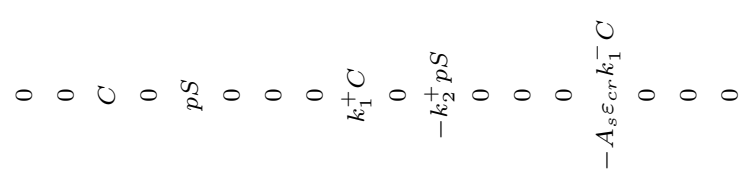

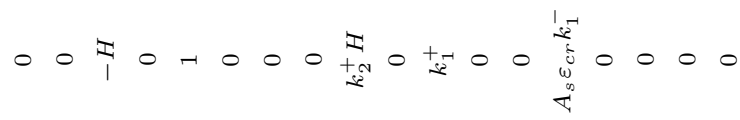

$$
\begin{aligned}
& 00 \pi 00000+4000000000
\end{aligned}
$$

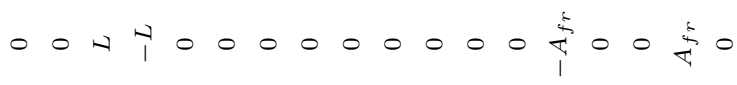

$$
\begin{aligned}
& 00 \overrightarrow{1} 00000000000000
\end{aligned}
$$

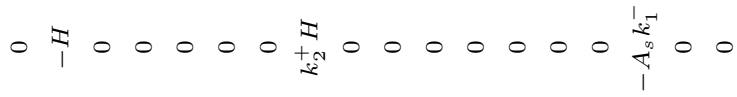

$$
\begin{aligned}
& 0-00000+10000000000
\end{aligned}
$$

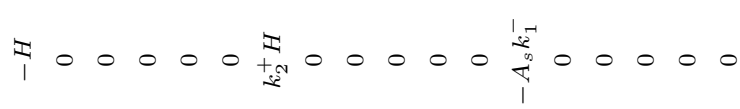

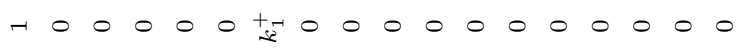


with:

$$
\begin{aligned}
& C=\cos (p H) \\
& S=\sin (p H) \\
& k_{1}^{+}=1-\exp (2 i k H) \\
& k_{2}^{+}=1+\exp (2 i k H) \\
& k_{1}^{-}=1-\exp (-2 i k H) \\
& k_{2}^{-}=1+\exp (-2 i k H) \\
& A_{s}=2 E_{s} t \\
& A_{f r}=2 E_{f r} e \\
& B_{f r}=\frac{4}{3} E_{f r} e^{3}
\end{aligned}
$$

\section{Appendix B. Comparison of the analytical and numerical critical displacements for the first 30 modes whatever the wave numbers}

\section{References}

[1] Hoff, N.J. and Mautner, S.E., Buckling of sandwich type panels, Journal of the Aeronautical Sciences 12 Issue 3 (1945) $285-297$.

[2] Allen, H.G., Analysis and Design of Structural Sandwich Panels (Pergamon, 1969).

[3] Benson, A.S. and Mayers, J., General instability and face wrinkling of sandwich plates: Unified theory and applications, AIAA Journal 5 Issue 4 (1967) 729-739.

[4] Douville, M.A. and Le Grognec, P., Exact analytical solutions for the local and global buckling of sandwich beam-columns under various loadings, International Journal of Solids and Structures 50 Issues 16-17 (2013) 2597-2609.

[5] López Jiménez, F. and Triantafyllidis, N., Buckling of rectangular and hexagonal honeycomb under combined axial compression and transverse shear, International Journal of Solids and Structures 50 Issue 24 (2013) 3934-3946.

[6] Lascoup, B., Aboura, Z., Khellil, K. and Benzeggagh, M.L., Prediction of out-of-plane behavior of stitched sandwich structure, Composites Part B: Engineering 43 Issue 8 (2012) 2915-2920.

[7] Liu, T., Deng, Z.C. and Lu, T.J., Analytical modeling and finite element simulation of the plastic collapse of sandwich beams with pin-reinforced foam cores, International Journal of Solids and Structures 45 Issues 18-19 (2008) 5127-5151.

[8] Lainé, C., Le Grognec, P., Comas Cardona, S. and Binetruy, C., Analytical, numerical and experimental study of the bifurcation and collapse behavior of a 3D reinforced sandwich structure under through-thickness compression, International Journal of Mechanical Sciences 67 (2013) 42-52.

[9] Zhang, G. and Latour, R.A., An analytical and numerical study of fiber microbuckling, Composites Science and Technology 51 Issue 1 (1994) 95-109.

[10] Wang, D. and Abdalla, M.M., Global and local buckling analysis of grid-stiffened composite panels, Composite Structures 119 (2015) 767-776.

[11] Combescure, C., Henry, P. and Elliott, R.S., Post-bifurcation and stability of a finitely strained hexagonal honeycomb subjected to equi-biaxial in-plane loading, International Journal of Solids and Structures 88-89 (2016) 296-318.

[12] Lascoup, B., Aboura, Z., Khellil, K. and Benzeggagh, M.L., On the mechanical effect of stitch addition in sandwich panel, Composites Science and Technology 66 Issue 10 (2006) 1385-1398.

[13] Zhang, G. and Latour, R.A., A three-dimensional micromechanical model of the compressive behavior of unidirectional FRP composites, Journal of Thermoplastic Composite Materials 10 Issue 2 (1997) 173-184.

[14] Boutin, C., Rallu, A. and Hans, S., Large scale modulation of high frequency waves in periodic elastic composites, Journal of the Mechanics and Physics of Solids $\mathbf{7 0}$ (2014) 362-381. 
[15] Bertoldi, K., Boyce, M.C., Deschanel, S., Prange, S.M. and Mullin, T., Mechanics of deformation-triggered pattern transformations and superelastic behavior in periodic elastomeric structures, Journal of the Mechanics and Physics of Solids 56 Issue 8 (2008) 2642-2668.

[16] Liu, J. and Bertoldi, K., Bloch wave approach for the analysis of sequential bifurcations in bilayer structures, Proceedings of the Royal Society A $\mathbf{4 7 1}$ Issue 2182 (2015)

[17] Gong, L., Kyriakides, S. and Triantafyllidis, N., On the stability of Kelvin cell foams under compressive loads, Journal of the Mechanics and Physics of Solids 53 Issue 4 (2005) 771-794.

[18] Wang, D., Abdalla, M.M. and Zhang, W., Buckling optimization design of curved stiffeners for grid-stiffened composite structures, Composite Structures 159 (2017) 656-666.

[19] Ning, X. and Pellegrino, S., Bloch wave buckling analysis of axially loaded periodic cylindrical shells, Computers 8 Structures 177 (2016) 114-125.

[20] Åberg, M. and Gudmunson, P., The usage of standard finite element codes for computation of dispersion relations in materials with periodic microstructure, Journal of the Acoustical Society of America 102 Issue 4 (1997) 2007-2013. 


\begin{tabular}{|c|c|c|c|c|c|}
\hline $\begin{array}{c}\text { Wave number } \\
\qquad k\end{array}$ & $\begin{array}{l}\text { Mode } \\
\text { number }\end{array}$ & $\begin{array}{l}\text { Critical displacement } \\
\text { - Analytical } u_{c r}^{a n}(m m)\end{array}$ & $\begin{array}{l}\text { Critical displacement } \\
\text { - Unit cell } u_{c r}^{U C}(\mathrm{~mm})\end{array}$ & $\begin{array}{c}\text { Critical displacement } \\
\text { - Complete column } u_{c r}^{C C}(\mathrm{~mm})\end{array}$ & $\begin{array}{l}\text { Relative error } \\
\frac{u_{c r}^{C C}-u_{c r}^{U C}}{u_{c r}^{U C}}(\%) \\
\end{array}$ \\
\hline \multirow{3}{*}{0} & $1^{s t}$ & 0.3567 & 0.359042 & 0.356810 & 0.622 \\
\hline & $2^{n d}$ & - & 0.377812 & - & - \\
\hline & $3^{r d}$ & - & 0.649882 & - & - \\
\hline \multirow{3}{*}{$\frac{\pi}{20 H}$} & $1^{s t}$ & 0.009395 & 0.010086 & 0.010125 & 0.387 \\
\hline & $2^{n d}$ & 0.3242 & 0.325999 & 0.324040 & 0.601 \\
\hline & $3^{r d}$ & 0.3299 & 0.329302 & 0.330370 & 0.324 \\
\hline \multirow{3}{*}{$\frac{2 \pi}{20 H}$} & $1^{s t}$ & 0.01208 & 0.012770 & 0.012814 & 0.345 \\
\hline & $2^{n d}$ & 0.2889 & 0.290404 & 0.288890 & 0.521 \\
\hline & $3^{r d}$ & 0.2975 & 0.298582 & 0.298130 & 0.151 \\
\hline \multirow{3}{*}{$\frac{3 \pi}{20 H}$} & $1^{\text {st }}$ & 0.01652 & 0.017203 & 0.017251 & 0.279 \\
\hline & $2^{n d}$ & 0.2539 & 0.256143 & 0.253950 & 0.856 \\
\hline & $3^{r d}$ & 0.2666 & 0.267668 & 0.267280 & 0.145 \\
\hline \multirow{3}{*}{$\frac{4 \pi}{20 H}$} & $1^{s t}$ & 0.02272 & 0.023398 & 0.023451 & 0.227 \\
\hline & $2^{n d}$ & 0.2197 & 0.217699 & 0.219800 & 0.965 \\
\hline & $3^{r d}$ & 0.2373 & 0.238105 & 0.238070 & 0.015 \\
\hline \multirow{3}{*}{$\frac{5 \pi}{20 H}$} & $1^{s t}$ & 0.03068 & 0.031352 & 0.031408 & 0.179 \\
\hline & $2^{n d}$ & 0.1871 & 0.186851 & 0.187260 & 0.219 \\
\hline & $3^{r d}$ & 0.2098 & 0.211118 & 0.210610 & 0.241 \\
\hline \multirow{3}{*}{$\frac{6 \pi}{20 H}$} & $1^{s t}$ & 0.04038 & 0.041048 & 0.041104 & 0.136 \\
\hline & $2^{n d}$ & 0.1574 & 0.158706 & 0.157490 & 0.766 \\
\hline & $3^{r d}$ & 0.1841 & 0.185267 & 0.184950 & 0.171 \\
\hline \multirow{3}{*}{$\frac{7 \pi}{20 H}$} & $1^{s t}$ & 0.0518 & 0.052449 & 0.052499 & 0.095 \\
\hline & $2^{n d}$ & 0.1318 & 0.132411 & 0.131950 & 0.348 \\
\hline & $3^{r d}$ & 0.1602 & 0.161312 & 0.161120 & 0.119 \\
\hline \multirow{3}{*}{$\frac{8 \pi}{20 H}$} & $1^{s t}$ & 0.06482 & 0.065442 & 0.065475 & 0.050 \\
\hline & $2^{\text {nd }}$ & 0.1121 & 0.112365 & 0.112190 & 0.156 \\
\hline & $3^{r d}$ & 0.1383 & 0.139350 & 0.139270 & 0.057 \\
\hline \multirow{3}{*}{$\frac{9 \pi}{20 H}$} & $1^{s t}$ & 0.07899 & 0.079512 & 0.079509 & 0.004 \\
\hline & $2^{\text {nd }}$ & 0.09955 & 0.099642 & 0.099659 & 0.017 \\
\hline & $3^{r d}$ & 0.1188 & 0.119896 & 0.119900 & 0.003 \\
\hline \multirow{3}{*}{$\frac{10 \pi}{20 H}$} & $1^{s t}$ & 0.08917 & 0.089397 & 0.089339 & 0.065 \\
\hline & $2^{\text {nd }}$ & - & 0.095417 & - & - \\
\hline & $3^{r d}$ & - & 0.108219 & - & - \\
\hline
\end{tabular}

Table B.2: Comparison between critical displacements obtained with the unit cell models and the entire column (the value of $k$ only refers to the unit cell calculations) 